\title{
Multi-loop integrand reduction techniques
}

\author{
Simon Badger ${ }^{* a}$, Hjalte Frellesvig ${ }^{b, c}$ and Yang Zhang ${ }^{c}$ \\ ${ }^{a}$ Theory Division, Physics Department, CERN, CH-1211 Geneva 23, Switzerland \\ ${ }^{b}$ Instituto Nazionale di Fisica Nucleare, Sezione di Roma, P.le Aldo Moro 2, 00185 Roma, Italy \\ ${ }^{c}$ Niels Bohr International Academy and Discovery Center, The Niels Bohr Institute, University of \\ Copenhagen, Blegdamsvej 17, DK-2100 Copenhagen, Denmark \\ E-mail: simon.badger@cern.ch
}

\begin{abstract}
We review recent progress in $D$-dimensional integrand reduction algorithms for two loop amplitudes and give examples of their application to non-planar maximal cuts of the five-point all-plus helicity amplitude in QCD.
\end{abstract}

Loops and Legs in Quantum Field Theory - LL 2014,

27 April - 2 May 2014

Weimar, Germany

\footnotetext{
${ }^{*}$ Speaker.
} 


\section{Introduction}

Multi-leg two-loop amplitudes are of potential importance for precision measurements in the coming years of high energy proton-proton collisions at the LHC. Improved understanding of IR subtraction schemes has led to considerable progress in the calculations of full NNLO QCD corrections for $2 \rightarrow 2$ processes. Recent examples of hadronic production of di-jets [1,2], $t \bar{t}[3,4]$, and $V V[5]$ are reviewed in these proceedings. The double virtual corrections required at this precision have mainly been obtained using Feynman diagrams together with integration-by-parts identities [6], but owing to the rapid growth in complexity this approach may not be sufficient to handle higher multiplicity processes and therefore new techniques are desirable.

Though Feynman diagram technology has been sufficient for a number of two-loop QCD amplitudes (those required for di-jets and Higgs plus jet production are arguably the most complicated amplitudes achieved with this approach [7-11]), on-shell approaches can avoid large intermediate steps and enable an efficient calculation of more complicated processes. Unitarity [12] and generalized unitarity [13] techniques have been successfully applied to two-loop QCD amplitudes for massless $2 \rightarrow 2$ processes [14-20]. In super-symmetric gauge and gravity theories these techniques are now a familiar technology, with the current state-of-the art computations being able to handle four and even five loops.

Encouraged by the high levels of automation achieved at NLO, there has been recent progress in extending unitarity, generalized unitarity and integrand reduction algorithms to allow a systematic algebraic approach to arbitrary loop amplitudes. The maximal unitarity approach proposed by Kosower and Larsen [21] builds upon the method of directly extracting of integral coefficients using generalized unitrarity cuts. Further developments in this direction are summarized in [22-29]. The integrand reduction algorithm developed by Ossola, Papadopoulos and Pittau (OPP) [30] has also been the focus of multi-loop extensions. Initial attempts to extend this method [31, 32] led to the proposal of the computational algebraic geometry method [33, 34], generalizing the integrand reduction algorithm systematically to all loop orders. A number of different examples have been considered within this framework [35-41]. Investigations into spinor integration methods at two-loops are also on-going [42].

It has been interesting to see algebraic geometry play an increasingly important role in understanding the details of these methods. Gröbner basis and polynomial division techniques allow the automation of the integrand reduction process, and the tool of primary decomposition characterizes the structure of the branches of the unitarity-cut solutions.

In these proceedings we review the $D$-dimensional formulation of the multi-loop integrand reduction method and present applications to maximal non-planar cuts of the five-gluon all-plus amplitude in QCD.

\section{A $D$-dimensional integrand reduction algorithm}

Our approach to $D$-dimensional integrand reduction has been developed during the compu- 
tation of the five-gluon all-plus helicity amplitude in QCD [41]. A summary of the approach has recently been presented in the proceedings of ACAT 2013 [43], so we will only give a very schematic overview here.

A $D=4-2 \varepsilon$ dimensional $L$ loop amplitude depending on a set of external momenta $\{p\}$ and internal momenta $\{k\}$ has the generic form:

$$
A_{n}^{(L),[D]}(\{p\})=\int \prod_{i=1}^{L} \frac{d^{D} k_{i}}{(2 \pi)^{D}} \frac{N(\{k\},\{p\})}{\prod_{l=1}^{L(L+9) / 2} D_{l}(\{k\},\{p\})},
$$

where $D_{l}$ are the denominators of the loop propagators. The master numerator function $N$ may be obtained from Feynman diagrams or by off-shell recursive techniques. Alternatively, $N$ can be written as the product of tree-level amplitudes when generalized unitarity cuts are applied to the propagators. The goal of the integrand reduction procedure is to write a loop amplitude in the form:

$$
A_{n}^{(L),[D]}(\{p\})=\int \prod_{i=1}^{L} \frac{d^{D} k_{i}}{(2 \pi)^{D}} \sum_{c=1}^{L(L+9) / 2} \sum_{T \in P_{c}} \frac{\Delta_{c ; T}\left(\left\{x_{i j}, \mu_{i j}\right\}\right)}{\prod_{l \in T} D_{l}(\{k\},\{p\})},
$$

where $x_{i j}$ and $\mu_{i j}$ are 4 and $-2 \varepsilon$ dimensional irreducible scalar products (ISPs) that must be identified. To achieve this we use the polynomial division algorithm proposed by Zhang [33] in $D$ dimensions:

1. Choose a maximal propagator topology from the list of un-computed topologies.

2. Choose a set of momenta, $v_{i}$, spanning the space of external momenta.

3. Change the propagator equations into scalar product variables $x_{i j}=\bar{k}_{i} \cdot v_{j}$ and $\mu_{i j}=k_{i}^{[-2 \varepsilon]}$. $k_{j}^{[-2 \varepsilon]}$ using the $4 \times 4$ Gram matrix, $G_{4}(v)$, to give a set of equations: $P_{\alpha}\left(x_{i j}, \mu_{i j}\right)$.

4. Separate the reducible scalar products from the irreducible scalar products using the linear parts of the propagator equations, $\langle P\rangle=\left\langle P_{\text {quadratic }}\right\rangle \cup\left\langle P_{\text {linear }}\right\rangle$.

5. Use polynomial division by the Gröbner basis of the ideal $\left\langle P_{\text {quadratic }}\right\rangle$ to define the integrand parametrization $\Delta_{T}=\sum_{i} c_{i} m_{i}\left(x_{i j}, \mu_{i j}\right)$ in terms of ISP monomials $m_{i}$.

6. Use primary decomposition of the algebraic variety $Z(P)$ to reduce the unitarity-cut solutions to irreducible branches. Solve the on-shell equations at each branch using an explicit parametrization of $k_{i}^{(s)}\left(\tau_{\alpha}\right)$.

7. Use $\Delta_{T}\left(k^{(s)}\right)=\sum d_{j} m_{j}^{\prime}\left(\tau_{\alpha}\right)=N\left(k^{(s)}\right)-\sum_{T} \Delta_{T}\left(k^{(s)}\right)=\prod A^{(0)}\left(k^{(s)}\right)-\sum_{T} \Delta_{T}\left(k^{(s)}\right)$ to compute the residues $d_{j}$ from the input (e.g. tree-level amplitudes, diagrams, etc). The sum runs over all (previously computed) topologies with higher number of propagators.

8. Solve the master system $M \cdot \vec{c}=\vec{d}$ for the coefficients $c_{i}$ where,

$$
\sum_{j} d_{j} m_{j}^{\prime}\left(\tau_{\alpha}\right)=\sum_{i} c_{i} m_{i}\left(x_{i j}\left(\tau_{\alpha}\right), \mu_{i j}\left(\tau_{\alpha}\right)\right)=\sum_{i, j} M_{j i} c_{i} m_{j}^{\prime}\left(\tau_{\alpha}\right)
$$


9. Go back to 1 until all topologies are computed.

Steps 2-5 above are implemented in Zhang's BASISDET Mathematica package [33].

There are a few important comments to make about this approach. Firstly there is an ambiguity as to which spanning basis vectors $v_{i}$ to choose and we notice in a number of examples that a good choice can result in a considerably more compact integrand representation. Secondly, step 6 can be efficiently performed using the primary decomposition algorithm implemented in MACAULAY2 [44]. In $D$-dimensions it is possible to prove that all propagator ideals are radical ideals and therefore admit exactly one on-shell solution branch. Thirdly, in step 5 we must provide an ISP ordering to define the polynomial division. Any choice will result in a valid integrand representation but also here the choice will affect the analytic form considerably. A feature of $D$-dimensional systems is that we may elect to remove monomials of the extra dimensional ISPs $\mu_{i j}$ in terms of monomials of the four dimensional ones $x_{i j}$. In certain circumstances this can result in a $D$-dimensional integrand basis which does not have a smooth limit onto the four dimensional case, and this is a feature which is highly undesirable and may require some additional manipulation after the polynomial division has been performed.

\section{Simplifying kinematics with momentum twistors}

The four-component momentum twistors, first introduced by Hodges [45], is $Z\left(p_{i}\right)=Z_{i}=$ $\left(\lambda_{i, a}, \mu_{i, a}\right)$ for a massless momentum $p_{i}$, where $\lambda_{i, a}$ are the two-component holomorphic Weyl spinors $(a=1,2) . \quad \mu_{i}$ are the dual variables which are used to construct the anti-holomorphic spinors:

$$
W_{i, \dot{a}}=\left(\tilde{\mu}_{i, \dot{a}}, \tilde{\lambda}_{i, \dot{a}}\right)=\frac{\varepsilon_{\dot{a}, b, c, d} Z_{i-1, b} Z_{i, c} Z_{i+1, d}}{\langle i-1, i\rangle\langle i, i+1\rangle} .
$$

While for four-point kinematics the minimal set of variables can be written in terms of Mandelstam invariants, the five-point case is complicated by a non-trivial Gram matrix identity relating the square of the trace operator including $\gamma_{5}$ to the invariants,

$$
\operatorname{tr}_{5}(1234)^{2}=16 \operatorname{det} G\left(\begin{array}{llll}
p_{1} & p_{2} & p_{3} & p_{4} \\
p_{1} & p_{2} & p_{3} & p_{4}
\end{array}\right) .
$$

This relation is satisfied explicitly after a transformation to the momentum twistor variables, $x_{i}$ :

$$
Z=\left(\begin{array}{ccccc}
1 & 0 & \frac{1}{x_{1}} & \frac{1}{x_{1}}+\frac{1}{x_{1} x_{2}} & \frac{1}{x_{1}}+\frac{1}{x_{1} x_{2}}+\frac{1}{x_{1} x_{2} x_{3}} \\
0 & 1 & 1 & 1 & 1 \\
0 & 0 & 0 & \frac{x_{4}}{x_{2}} & 1 \\
0 & 0 & 1 & 1 & 1-\frac{x_{5}}{x_{4}}
\end{array}\right) .
$$

The variables $x_{i}$ can be expressed in terms of the usual spinor products and kinematic invariants as:

$$
\begin{array}{ll}
x_{1}=s_{12}, & x_{4}=\frac{s_{23}}{s_{12}}, \\
x_{2}=-\frac{\langle 23\rangle\langle 41\rangle}{\langle 12\rangle\langle 34\rangle}, & x_{3}=-\frac{\langle 34\rangle\langle 51\rangle}{\langle 13\rangle\langle 45\rangle} .
\end{array}
$$




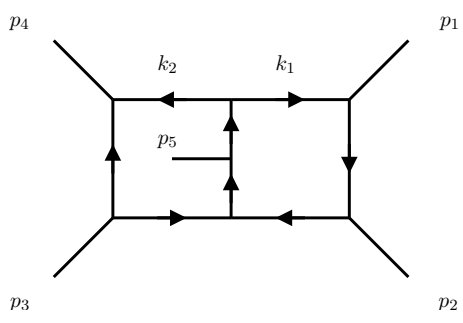

(a) The topology (332).

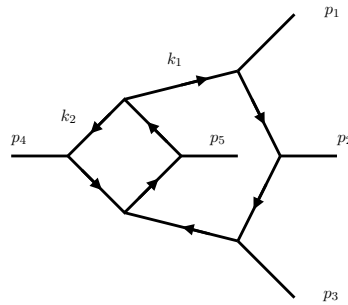

(b) The topology (422).

Figure 1: Maximal cut non-planar topologies for five-point amplitudes.

By converting expressions to these variables during step 7 of the algorithm, we can automatically express the Feynman diagram input as compact analytic expressions for the $d_{j}$ coefficients.

\section{Example: non-planar maximal cuts of five-gluon all-plus helicity amplitude}

There are two independent non-planar topologies for massless five-point amplitudes as depicted in figure 1. We label each topology by the number of propagators along each of the three loop momentum branches $k_{1}, k_{2}$ and $k_{1}+k_{2}$ so topology (a) is 332 and (b) is 422 .

\subsection{The 332 topology}

This topology is defined by the propagators:

$$
\left\{k_{1}, k_{1}-p_{1}, k_{1}-p_{1}-p_{2}, k_{2}, k_{2}-p_{4}, k_{2}-p_{3}-p_{4}, k_{1}+k_{2}, k_{1}+k_{2}+p_{5}\right\}
$$

A good choice for the spanning basis turns out to be $v=\{5,1,4,2\}$ which, using a lexicographic ordering, gives a generic basis of 82 monomials under the renormalization constraints $\{5,5,6\}$. The BASISDET code is rather simple and reads:

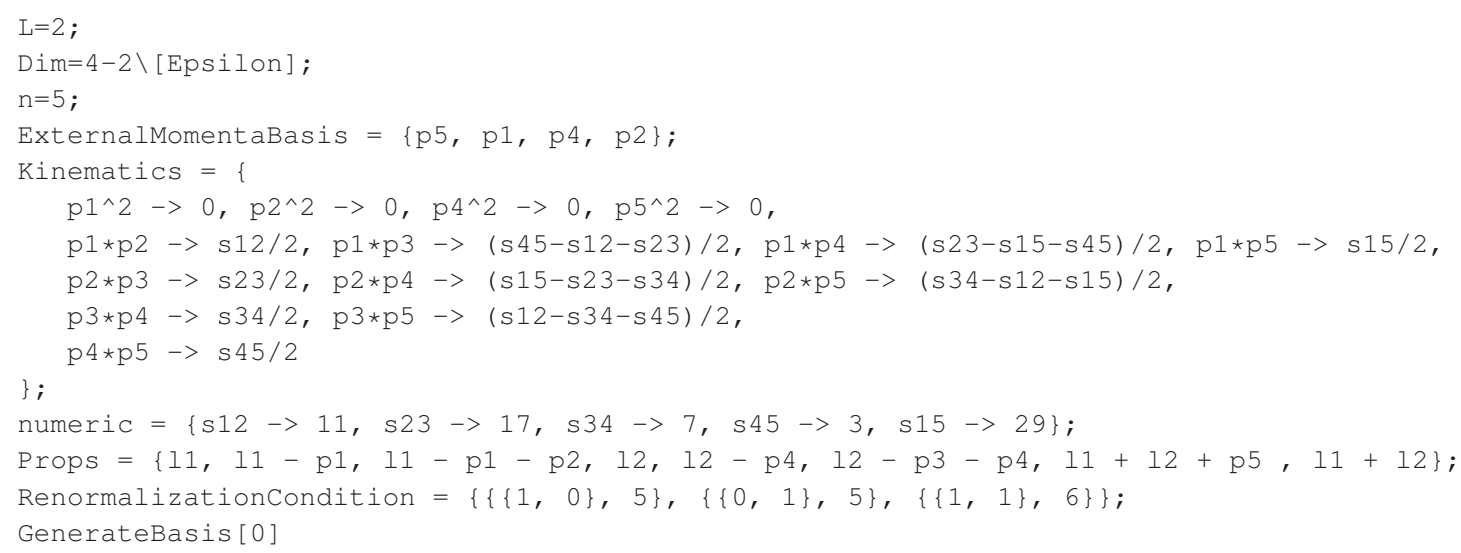


The on-shell loop momenta are conveniently parametrized in terms of 3 free variables:

$$
\begin{aligned}
& \bar{k}_{1}^{\mu}=p_{1}^{\mu}+\tau_{1} \frac{[13]}{2[23]}\left\langle 1\left|\gamma^{\mu}\right| 2\right]+\tau_{2} \frac{\langle 13\rangle}{2\langle 23\rangle}\left\langle 2\left|\gamma^{\mu}\right| 1\right], \\
& \bar{k}_{2}^{\mu}=p_{4}^{\mu}+\tau_{3} \frac{\langle 14\rangle}{2\langle 13\rangle}\left\langle 3\left|\gamma^{\mu}\right| 4\right]+\beta \frac{\langle 13\rangle}{2\langle 14\rangle}\left\langle 4\left|\gamma^{\mu}\right| 3\right],
\end{aligned}
$$

where

$$
\beta=\frac{-s_{14}}{\operatorname{tr}_{-}(1354)}\left(s_{15}+s_{45}+\frac{1}{s_{23}}\left(\tau_{1} \operatorname{tr}_{-}(1523)+\tau_{2} \operatorname{tr}_{+}(1523)\right)+\frac{1}{s_{13}} \tau_{3} \operatorname{tr}_{-}(1453)\right) .
$$

We can then construct a linear system of $82 \times 83$ to fit the integrand coefficients. In the case of the 5-gluon all-plus amplitude the situation is much simpler than this general parametrization suggests, and we find that only three independent ISP monomials are present with a structure similar to that of the planar case [41]. The result is:

$$
\begin{aligned}
\Delta_{8 ; 332}\left(1^{+}, 2^{+}, 3^{+}, 4^{+}, 5^{+}\right) & =\frac{2 i F_{1} s_{12} s_{34}}{\langle 12\rangle\langle 23\rangle\langle 34\rangle\langle 45\rangle\langle 51\rangle \operatorname{tr}_{5}}\left(c_{1} k_{1} \cdot p_{4}+c_{2} k_{2} \cdot p_{1}+c_{3} k_{1} \cdot p_{5}\right), \\
c_{1} & =-s_{15} \operatorname{tr}_{-}(2345) \\
c_{2} & =s_{45} \operatorname{tr}_{-}(2351) \\
c_{3} & =s_{23} s_{45} s_{15}-s_{15} \operatorname{tr}_{-}(2345)-s_{45} \operatorname{tr}_{-}(2351) \\
F_{1} & =\left(D_{s}-2\right)\left(\mu_{11} \mu_{22}+\mu_{22} \mu_{33}+\mu_{33} \mu_{11}\right)+4\left(\mu_{12}^{2}-4 \mu_{11} \mu_{22}\right),
\end{aligned}
$$

where $\mu_{33}=\mu_{11}+\mu_{12}+\mu_{22}$, and $D_{s}=g_{\mu}^{\mu}$ is the spin dimension.

\subsection{The 422 topology}

This topology is defined by the propagators:

$$
\left\{k_{1}, k_{1}-p_{1}, k_{1}-p_{1}-p_{2}, k_{1}-p_{1}-p_{2}-p_{3}, k_{2}, k_{2}-p_{4}, k_{1}+k_{2}, k_{1}+k_{2}+p_{5}\right\} \text {, }
$$

and again in this case we use $v=\{5,1,4,2\}$. To make the four-dimensional limit of the integrand representation manifest we must make some replacements to the monomial list. One possible change is:

$$
\begin{aligned}
& x_{22}^{4} \rightarrow x_{22}^{4} \mu_{22}, \quad x_{22}^{3} \rightarrow x_{22}^{3} \mu_{22}, \quad x_{22}^{2} \rightarrow x_{22}^{2} \mu_{11}, \\
& x_{24} x_{22}^{3} \rightarrow x_{24} x_{22}^{3} \mu_{22}, \quad x_{24} x_{22}^{2} \rightarrow x_{24} x_{22}^{2} \mu_{22}, \quad x_{24} x_{22} \rightarrow x_{24} x_{22} \mu_{11} \text {, } \\
& x_{21} x_{22} \rightarrow x_{21} x_{22} \mu_{22}^{2} \text {. }
\end{aligned}
$$

The on-shell solution can be parametrized as:

$$
\begin{aligned}
& \bar{k}_{1}^{\mu}=p_{1}^{\mu}+\tau_{1} \frac{\langle 23\rangle}{2\langle 13\rangle}\left\langle 1\left|\gamma^{\mu}\right| 2\right]+\left(1-\tau_{1}\right) \frac{[23]}{2[13]}\left\langle 2\left|\gamma^{\mu}\right| 1\right], \\
& \bar{k}_{2}^{\mu}=\beta p_{4}^{\mu}+\tau_{2} \frac{\langle 15\rangle}{2\langle 14\rangle}\left\langle 4\left|\gamma^{\mu}\right| 5\right]+\tau_{3} \frac{[15]}{2[14]}\left\langle 5\left|\gamma^{\mu}\right| 4\right],
\end{aligned}
$$


where

$$
\beta=-\frac{1}{s_{45} s_{13}}\left(s_{13} s_{15}+\tau_{1} \operatorname{tr}_{-}(1523)+\left(1-\tau_{1}\right) \operatorname{tr}_{+}(1523)\right) .
$$

Inverting the resulting $65 \times 76$ linear system gives a simple representation for the all-plus helicity configuration:

$$
\begin{aligned}
\Delta_{8 ; 422} & =\frac{i F_{1} s_{12} s_{23} s_{45}}{\langle 12\rangle\langle 23\rangle\langle 34\rangle\langle 45\rangle\langle 51\rangle \operatorname{tr}_{5}}\left(c_{0}+2 c_{1} k_{2} \cdot p_{5}\right), \\
c_{0} & =s_{15} s_{34} s_{45} \\
c_{1} & =-\operatorname{tr}_{+}(1345) .
\end{aligned}
$$

where $F_{1}$ refers to eq. (4.9).

As observed in the case of the planar topologies [41], these non-planar contributions also have compact representations for the all-plus configuration. Though more general QCD helicity amplitudes will be significantly more complicated, we hope that the techniques presented here will help to make these computations possible in the near future.

\section{References}

[1] A. G.-D. Ridder, T. Gehrmann, E. Glover, and J. Pires Phys. Rev. Lett. 110 (2013) 162003, [1301.7310].

[2] J. Currie, A. Gehrmann-De Ridder, E. Glover, and J. Pires JHEP 1401 (2014) 110, [1310 . 3993].

[3] G. Abelof, A. Gehrmann-De Ridder, P. Maierhöfer and S. Pozzorini 1404.6493.

[4] M. Czakon, P. Fiedler, and A. Mitov Phys. Rev. Lett. 110 (2013) 252004, [1303. 6254].

[5] F. Cascioli, T. Gehrmann, M. Grazzini,S. Kallweit, P. Maierhöfer and others 1405.2219.

[6] K. Chetyrkin and F. Tkachov Nucl. Phys. B192 (1981) 159-204.

[7] C. Anastasiou, E. Glover, C. Oleari, and M. Tejeda-Yeomans Nucl. Phys. B601 (2001) 318-340, [hep-ph/0010212].

[8] C. Anastasiou, E. Glover, C. Oleari, and M. Tejeda-Yeomans Nucl. Phys. B601 (2001) 341-360, [hep-ph/0011094].

[9] C. Anastasiou, E. Glover, C. Oleari, and M. Tejeda-Yeomans Nucl. Phys. B605 (2001) 486-516, [hep-ph/0101304].

[10] E. Glover, C. Oleari, and M. Tejeda-Yeomans Nucl. Phys. B605 (2001) 467-485, [hep-ph/0102201].

[11] T. Gehrmann, M. Jaquier, E. Glover, and A. Koukoutsakis JHEP 1202 (2012) 056, [1112 . 3554 ].

[12] Z. Bern, L. J. Dixon, D. C. Dunbar, and D. A. Kosower Nucl. Phys. B425 (1994) 217-260, [hep-ph/9403226].

[13] R. Britto, F. Cachazo, and B. Feng Nucl. Phys. B725 (2005) 275-305, [hep-th/ 0412103 ].

[14] Z. Bern, L. J. Dixon, and D. Kosower JHEP 0001 (2000) 027, [hep-ph/ 0001001 ]. 
[15] Z. Bern, L. J. Dixon, and A. Ghinculov Phys. Rev. D63 (2001) 053007, [hep-ph/ 0010075 ].

[16] Z. Bern, A. De Freitas, and L. J. Dixon JHEP 0109 (2001) 037, [hep-ph/ 0109078 ].

[17] Z. Bern, A. De Freitas, L. J. Dixon, A. Ghinculov, and H. Wong JHEP 0111 (2001) 031, [hep-ph/0109079].

[18] Z. Bern, A. De Freitas, and L. J. Dixon JHEP 0203 (2002) 018, [hep-ph/ 0201161 ].

[19] Z. Bern, A. De Freitas, L. J. Dixon, and H. Wong Phys. Rev. D66 (2002) 085002 , [hep-ph/0202271].

[20] Z. Bern, A. De Freitas, and L. J. Dixon JHEP 0306 (2003) 028, [hep-ph/ 0304168 ].

[21] D. A. Kosower and K. J. Larsen Phys. Rev. D85 (2012) 045017, [1 108 . 1180 ].

[22] H. Johansson, D. A. Kosower, and K. J. Larsen Phys. Rev. D87 (2013) 025030, [1208 . 1754 ].

[23] H. Johansson, D. A. Kosower, and K. J. Larsen 1308.4632.

[24] S. Caron-Huot and K. J. Larsen JHEP 1210 (2012) 026, [120 5 . 0801 ].

[25] K. J. Larsen Phys. Rev. D86 (2012) 085032, [1205. 02 97$].$

[26] M. Søgaard JHEP 1309 (2013) 116, [1306.1496].

[27] M. Søgaard and Y. Zhang JHEP 1312 (2013) 008, [1310 . 600 6].

[28] M. Søgaard and Y. Zhang 1403.2463.

[29] M. Søgaard and Y. Zhang 1406.5044.

[30] G. Ossola, C. G. Papadopoulos, and R. Pittau Nucl. Phys. B763 (2007) 147-169, [hep-ph/0609007].

[31] P. Mastrolia and G. Ossola JHEP 1111 (2011) 014, [1107.6041].

[32] S. Badger, H. Frellesvig, and Y. Zhang JHEP 1204 (2012) 055, [1202 . 2019 ].

[33] Y. Zhang JHEP 1209 (2012) 042, [1205. 5707].

[34] P. Mastrolia, E. Mirabella, G. Ossola, and T. Peraro Phys. Lett. B718 (2012) 173-177, [12 05.7087 ].

[35] S. Badger, H. Frellesvig, and Y. Zhang JHEP 1208 (2012) 065, [1207 . 2976 ].

[36] P. Mastrolia, E. Mirabella, G. Ossola, and T. Peraro Phys. Rev. D87 (2013) 085026, [1209. 4319 ].

[37] R. H. Kleiss, I. Malamos, C. G. Papadopoulos, and R. Verheyen JHEP 1212 (2012) 038, [1206.4180].

[38] B. Feng and R. Huang JHEP 1302 (2013) 117, [1209. 3747].

[39] P. Mastrolia, E. Mirabella, G. Ossola, and T. Peraro Phys. Lett. B727 (2013) 532-535, [1307 . 5832 ].

[40] R. Huang and Y. Zhang JHEP 1304 (2013) 080, [1302 . 1023].

[41] S. Badger, H. Frellesvig, and Y. Zhang JHEP 1312 (2013) 045, [1310 . 1051 ].

[42] B. Feng, J. Zhen, R. Huang, and K. Zhou 1401.6766.

[43] S. Badger, H. Frellesvig, and Y. Zhang J.Phys. Conf. Ser. 523 (2014) 012061, [1310 . 4445$].$

[44] D. R. Grayson and M. E. Stillman, MACAULAY2, a software system for research in algebraic geometry. Available at http://www.math.uiuc.edu/Macaulay2/.

[45] A. Hodges JHEP 1305 (2013) 135, [0 905 . 1473]. 